\title{
Designated Verification of Digital Watermark for Network Based Image Distribution ${ }^{\star}$
}

\author{
Hyung-Woo Lee ${ }^{1}$ and Im-Yeong Lee ${ }^{2}$ \\ 1 Dept. of Software, Hanshin University, Osan, Kyunggi, Korea, 447-791 \\ hwlee@hanshin.ac.kr \\ http://infocom. cheonan.ac.kr/ ${ }^{\mathrm{h} w l e e / i n d e x . h t m l}$ \\ 2 Div. of Information Technology Engineering, Soonchunhyang University, \\ Chungnam, Korea, 336-745 \\ imylee@sch.ac.kr
}

\begin{abstract}
Digital watermark have been proposed as the means for copyright protection on network based digital image distribution framework. However, there are some problems in existing digital watermarking mechanism as the rightful ownership cannot be resolved by current watermarking schemes alone. Specially, some one can provide counterfeited watermark which can be performed on a watermarked image to allow multiple claims of rightful ownerships. Therefore, we can use undeniable verification scheme in the watermarking process. And using designated verifier proofing schemes, watermark can be verified by commitment methods. The confirmer of a watermark can sure that only the intended verifier can be convinced about the validity of the watermark.
\end{abstract}

\section{Introduction}

An unlimited number of perfect copies can be illegally produced is a serious threat to the rights of content owners. The primary tool available to help protect content owners's rights is to use a encryption module. However, after receipt and subsequent decryption, the data is no longer protected and is in the clear.

Watermarking compliments encryption. A digital watermark is a piece of information that is hidden directly in media content, in such a way that it is imperceptible to a human observer, but easily detected by a computer. The principal advantage of this is that the content is inseparable from the watermark. This makes watermarks suitable for several applications, including:

- Signatures: The watermark identifies the owner of the content. This information can be used by a potential user to obtain legal rights to copy or publish the content from the contact owner.

- Fingerprinting: Watermarks can also be used to identify the content buyers. This may potentially assist in tracing the source of illegal copies. Each copies holds a watermark that uniquely identifies the player in every movie that is played.

* This work was supported by grant No. (R05-2001-000-01468-0) from the Basic Research Program of the Korea Science \& Engineering Foundation. 
- Authentication: The watermark encodes information required to determine that the content is authentic. If the watermark is present, and properly matches the content, the user of the content can be assured that it has not been altered since the watermark was inserted.

- Copy control: The watermark contains information about the rules of usage and copying which the content owner wishes to enforce.

- Secret communication: The embedded signal is used to transmit secret information from one person (or computer) to another, without anyone along the way knowing that this information is being sent. This is the classical application of steganography - the hiding of one piece of information within another.

A digital watermark is a signal added to digital data such that it could be used (1) to identify source of the data or uniquely establish ownership, (2) to identify its intended recipient, and (3) to check if the data has been tampered with. Within each class of applications, there could be variations on the requirement of the watermarking scheme[1]. Therefore, digital watermarks have been proposed as the means for copyright protection of multimedia data on network based digital image distribution structure. However, there are some problems in existing digital watermarking mechanism as the rightful ownerships cannot be resolved by current watermarking schemes alone. Specially, some one can provide counterfeited watermark which can be performed on a watermarked image to allow multiple claims of rightful ownerships[2].

There are several attacks in current digital watermark system. In first case, we can suppose that Alice and Bob use the same digital watermarking technique to watermark and there is one unique decoding scheme to extract the labels embedded in the images. In this case, if a watermarked image contains both Alice and Bob's signatures, how can we decide the originality of this marked image? And we also think another scenario. Let's suppose that Alice and Bob use different watermarking techniques. And Given a watermarked image, Alice can take this image and decode the label using her decoding scheme. Similarly Bob can perform the label extraction process with his decoding scheme. If Alice's decoder indicates that the image belongs to her while Bob's decoder indicates that it is his image, then how can we decide the ownership of this marked image? In these two cases, rightful ownership cannot be resolved by invisible watermarks alone. They present in detail a counterfeit watermarking scheme that allows multiple claims of ownerships.

Undeniable watermark differ from ordinary digital watermark in that the verifier should be unable to distinguish between valid and invalid watermark. But, original watermarker can change it into correct one. If the message in is copyright owner's information, the scheme can be non-invertible. And we can also introduce zero-knowledge based watermarking mechanism. Using zero-knowledge protocols[3], watermarks can be made public enough to be detected yet private enough to be unremovable. i.e., watermarking scheme in which the watermark's presence can be verified without ever revealing enough information to remove the mark. 
We first present the overall digital watermark mechanism and review the existing model in section 2. We then consider re-watermarking attack and its possible solution in section 3. And then we propose designated verification process with commitment scheme in section 4 . Furthermore, we discuss the proof of ownership in section 5 and conclude this study with the consideration of the future works in final section.

\section{Network Based Digital Image Distribution}

\subsection{Watermark: Function and Mechanism}

In general, there are two types of digital watermarks addressed in existing literature, (1) visible and (2) invisible watermarks. These watermarking schemes are designed mainly for two purposes : copyright protection and data authentication. In this paper we shall focus on the applicability of watermarking techniques for one instance of ownership verification, identification of an content's rightful owner(s)[2].

The watermarks embedded in an image have to be recoverable, despite intentional or unintentional modification of the image. They must also be invulnerable to deliberate attempts to forge, remove, or invalidate watermarks. Unfortunately, many of existing schemes have not addressed the ends of invisible watermarking schemes. They instead focused on the means to label an image invisibly and the robustness of the inserted labels against malicious attacks. Without any verification techniques or specification of certain requirements for digital watermark, anyone can claim ownership of any image by using his/her own verification methods. Therefore, it is crucial that watermark for copyright protection be able to be undeniably verified with original watermark embedder's verification.

We define in general terms the process of watermark insertion into an image and the use of undeniable watermarks to determine the ownership of a watermarked image. A watermark is inserted into an image, and the decoding process by which a watermark is recovered and then compared to the inserted watermark. We use to denote $I$ an image, $W$ a watermark consisting of a sequence of ownership labels $W=\left\{w_{1}, w_{2}, \ldots, w_{n}\right\}$ and the watermarked image $I^{W} . E$ is an encoder function if it takes an image $I$ and a watermark $W$ and generates a new image which is called the watermarked image $I^{W}$ i.e., $E(I, W)=I^{W}$.

We can construct both encoding and corresponding decoding processes. One common approach is to embed a watermark $W=\left\{w_{1}, w_{2}, \ldots, w_{n}\right\}$ into a set of derived features $F(I)=\left\{f_{1}(I), f_{2}(I), \ldots, f_{n}(I)\right\}$. The embedding process is achieved by an insertion $\oplus$ operation, $f_{i}^{*}=f_{i} \oplus w_{i}$. The insertion operation has an inverse operation, namely the extraction operation, $\ominus$ that we denote by i.e., $f_{i}^{*} \ominus f_{i}=w_{i}$. 


\subsection{Watermark Verification}

A watermarking scheme with detection consists of four polynomial-time algorithms $G E N_{K E Y}, G E N_{W}$, embed function $E$, and detection function $D$. $G E N_{K E Y}$ and $G E N_{W}$ are probabilistic and generate a key-pair $\left(k_{e m b}, k_{d e t}\right)$ and a watermark $W$. The algorithm $E\left(I, W, k_{e m b}\right)$ imperceptibly embeds the watermark $W$ into the cover-data $I$, using the key $k_{e m b}$. This results in stego-data $I^{W}$ (watermarked version of $I)$. The algorithm $D\left(I^{\prime}, W, I, k_{\text {det }}\right.$ ) returns a boolean value, which states whether the cover data $I^{\prime}$ contains the watermark $W$ relative to the reference data $I$, using key $k_{\text {det }}$. A symmetric watermarking scheme needs the same key $k_{w}$ ) for detection as for embedding. Unkeyed watermarking schemes need no key for embedding or detection. Watermarking schemes whose detect algorithms $D$ do not require the input of reference data $I$ are called blind, in contrast to non-blind schemes.

We can consider three watermark mechanisms for digital data $D^{\prime}$, which are the (1) watermark $(W)$ casting mechanism, (2) signature $\left(D^{*}\right)$ signing mechanism, and (3) signature hiding and verification mechanism. In this Fig. 2, we can easily understand two mechanisms among these three mechanism. We can embed some mark $W$ on digital data $D^{\prime}$. So, it is the common watermark embedding and verification(recovery) mechanism(mark casting mechanism). The other one is the cryptographic mechanism as we can attach digital signature $D^{*}$ on digital data $D^{\prime}$ for authentication(signing mechanism). From these two mechanism, we can consider new hybrid mechanism(signature hiding and verification mechanism). As we can think signature as its watermark information, we can hide this signature on its digital data for verifying ownership and authentication. So, we can construct hybrid mechanism on digital watermark verification and authentication system.

\section{Attack and Its Solution}

\subsection{Re-watermarking Attack}

In common case, we can assume that there is only one watermark in image for ownership dispute. It is not unreasonable to assume that multiple watermarked versions of the same image may indeed exist. But, if there are multiple watermark in one image, how can we decide the original watermark? And if we cannot find out which so-called original image is the true original, how can we decide which watermarked version of an image is the truely watermarked version in circulation? Bob is free to create as many watermarked versions with his own watermarks embedded after he has reverse-engineered a counterfeit original from Alice's watermarked version. To counter the claims, Bob has to prove the presence of his watermark in these watermarked images and the watermarked version of Alice, in addition to the presence of his watermark in Alice's original. S. Craver solves this problems as follows. He define non-invertible watermark and advanced non-quasi-invertible watermark scheme. By using this concepts, 
he can do prevent multiple watermark attacks. But there are some unsolved problems in above mechanism.

\subsection{Solution: Undeniable Verification}

Multiple watermarking problem must be solved easily if we consider it in verification process. In case of several attack proposed by S. Craver, we only review its robustness on verification module. Therefore, we can solve proposed attack problems from different directions compared with S. Craver's mechanism. In proposed system, if there are multiple watermarked image, the owner pseudoowner:prover) can cooperate its verification process. So, in case that the examined watermark ( pseudo-watermark) can also be matched with the verifier's input message on true-watermark, we can trust the pseudo-owner as really true-owner.

\subsection{Undeniable Property of Digital Watermark}

Digital watermarks are verified as authentic by anyone using the verification process. However, this self-verification property is unsuitable from many applications such as watermark based commercial digital contents distribution system.

The validity or invalidity of an undeniable watermark can be ascertained by conducting a verification process with original watermarker. If a confirmation process is needed, the cooperating original watermarker gives exponentially-high certainty to the verifier that the watermark does correspond to the legal one. We can use undeniable scheme in the watermarking process. This is because the confidence measure relies on the fact that the watermark vector can be later extracted, and using a commitment mechanism make designated verifier can certificate the legal owner on embedded watermark information.

Multimedia image company could embed the watermark which they signed using an undeniable scheme. Only someone who had directly purchased the image from that company could verify the watermark and be certain that the image were right. Undeniable watermark verification could also be useful in any situation in which an individual wishes to sign a data anonymously.

\subsection{New Verification Mechanism}

We can propose a new verification mechanism by using designated verification mechanism as Fig. 1. In first case, the original watermarker can embed his own secret(true watermark) on cover data and generate watermarked data. And it is distrubuted to the buyer by using network protocol. And then the authorized user can buy or get this data. However, the attacker can also multiply re-watermark his own fabricated watermark on distributed data. In this case, illegal data can be generated by attacker. So, if this data will be used by unauthorized user, we must verify and proof its legality and correctness on copied data. Therefore, we can suggest a new designated verification mechanism on watermarked data as the authorized user can verify its correctness by doing a undeniable-style proof with original watermarker. 


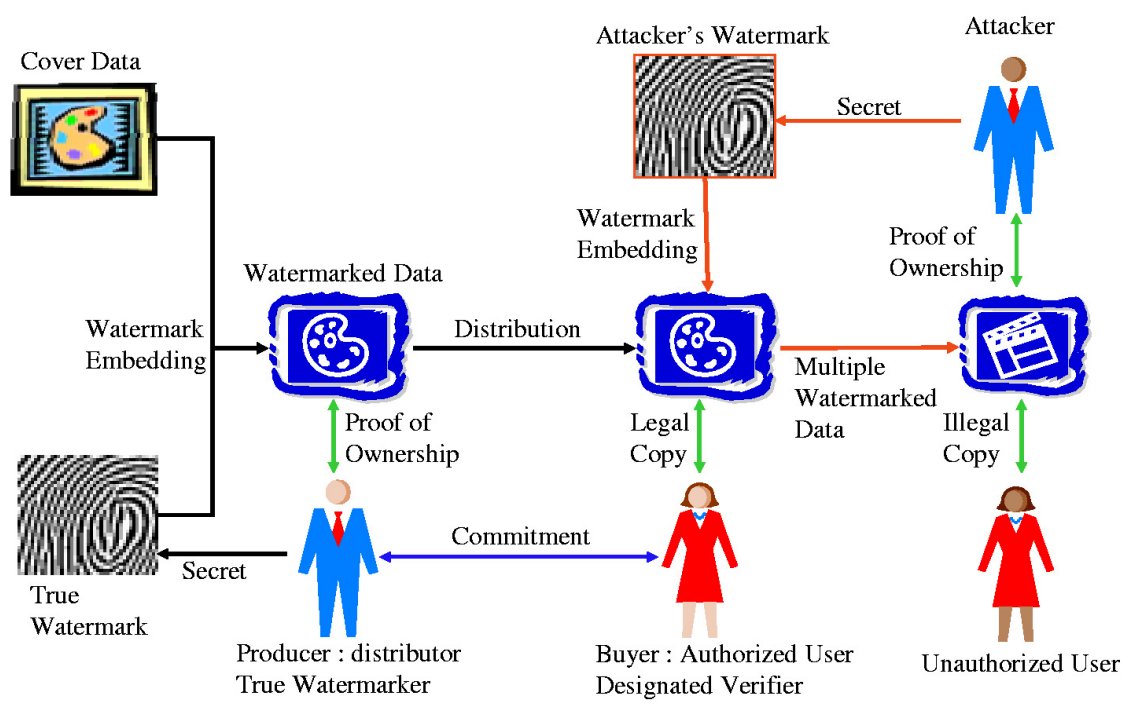

Fig. 1. New Watermark Verification Mechanism.

\section{Designated Verification for Network Based Image Distribution}

When a content owner sells a digital products, he only want to paying customers to be able to verify the validity of these image. Therefore, the designated verifier mechanism resolves this problems. We can designate a verifier when we ascertain that nobody but this participant can be convinded by the proof. With a small change in the confirmation protocol, we can designate verifier and demonstrate watermark verification scheme.

We can define designated verifier as follows. Let $\left(P_{A}, P_{B}\right)$ be a protocol for Alice to prove the truth of the watermark to Bob. In this case, we can say that Bob is a designated verified if the following is true: For any protocol $\left(P_{A}, P_{B}^{\prime}, P_{C}\right)$ involving Alice, Bob and Cindy, in which Bob proves the truth of $v$ to Cindy, there is another protocol $\left(P_{B}^{\prime \prime}, P_{C}\right)$ such that Bob can perform the calculations of $P_{B}^{\prime \prime}$ and Cindy cannot distinguish transcripts of $\left(P_{A}, P_{B}^{\prime}, P_{C}\right)$ from those of $\left(P_{B}^{\prime \prime}, P_{C}\right)$.

\subsection{Commitment Scheme[4]}

A commitment scheme (com,open) for the message space $M$ and commitment space $C$ consists of a two-party protocol com to commit to a value $m \in M$ and a protocol open that opens a commitment. A commitment to a value $m$ is denoted by $\operatorname{com}\left(m, \operatorname{par}_{\text {com }}\right)$ where $\operatorname{par}_{\text {com }}$ stands for all public parameters needed to compute the commitment value. To open a commitment com the committer 
runs the protocol open $\left(c o m, p a r_{c o m}, s k_{c o m}\right)$ where $s k_{c o m}$ is the secret opening information of the committer.

The security requirements are the binding(committing) and hiding(secrecy) properties. The first one requires that a dishonest committer cannot open a commitment to another message $m^{\prime}$ than the one to which he committed and the second one requires that the commitment does not reveal any information about the message $m$ to the verifier.

We require that the commitment scheme has following homomorphic property: Let $\operatorname{com}\left(m_{1}\right)$ and $\operatorname{com}\left(m_{2}\right)$ be commitments to arbitrary messages $m_{1}, m_{2} \in M$. Then the committer can open $\operatorname{com}\left(m_{1}\right) * \operatorname{com}\left(m_{2}\right)$ to $m_{1}+m_{2}$ without revealing additional information about the image of $\operatorname{com}\left(m_{1}\right)$ and $\operatorname{com}\left(m_{2}\right)$. So, we can combine these commitment mechanism on digital watermark verification procedures, if additional trap-door one-way properties are hold.

\subsection{Trap-Door Commitment}

For hybrid this commitment mechanism on designated verification technique, we consider trap-door primitives, in which we say that $c$ is a trap-door commitment scheme if and only if :

1. no polynomial-time machine can, given $y_{i}$, find a multiple $\left(\omega_{1}, r_{1}\right),\left(\omega_{2}, r_{2}\right)$ such that $c\left(y_{i}, \omega_{1}, r_{1}\right)=c\left(y_{i}, \omega_{2}, r_{2}\right)$.

2. no polynomial-time machine can, given $y_{i}$ and $c\left(y_{i}, \omega, r\right)$, output $\omega$.

3. there is a polynomial-time machine that given any quadruple $\left(x_{i}, w_{1}, r_{1}, \omega_{2}\right)$ in the set of possible quadruples finds $r_{2}$ such that $c\left(y_{i}, \omega_{1}, r_{1}\right)=c\left(y_{i}, \omega_{2}, r_{2}\right)$ for the public key $y_{i}$ corresponding to the secret key $x_{i}$.

Using this definitions [4], we can review simple trap-door commitment scheme.

1. Secret extracting(decryption) scheme from watermarked data : $D(\cdot)$.

2. Public embedding(encryption) scheme for watermarked data : $E(\cdot)$.

3. Value to commit to : $\omega \in \operatorname{Range}(E)$.

4. Commitment : Alice randomly selects $r \in \operatorname{Range}(E)$. And Alice calculates a commitment $c=E(\omega) \oplus E(r)$, where $\oplus$ is a combiner such as $X O R$.

5. Decommitment : Alice sends Bob $(\omega, r)$.

It is a trap-door commitment scheme if arbitrary collisions $\left(\omega_{1}, r_{1}\right),\left(\omega_{1}, r_{1}\right)$ such that $E\left(\omega_{1}\right) \oplus E\left(r_{1}\right)=E\left(\omega_{2}\right) \oplus E\left(r_{2}\right)$ can be found if and only if $D(\cdot)$ is known. By using this mechanism, we can show hot to change the normal watermark verification scheme for undeniable verification. Using this scheme, we can apply it into designated verification for undeniable watermark system.

\subsection{Undeniable Verification with Commitment}

Let (com, open) be a secure commitment scheme. A watermark verification protocol for the $\left(G E N_{K E Y}, G E N_{W}, E, D\right)$ is a undeniable proof protocol between 
a prover $P$ and a verifier $V$ : The common protocol input of $P$ and $V$ is the stego-data $I^{W}, \operatorname{com}(I), \operatorname{com}(W), \operatorname{com}\left(k_{w}\right)$, i.e., commitments on the watermark, the reference data and the detection key respectively, as well as the public parameters $\operatorname{par}_{\text {com }}=\left(\right.$ par $_{\text {com }}^{W}$, par $_{\text {com }}^{I}$, par $\left._{\text {com }}^{k_{w}}\right)$ of these commitments. The private input of the prover is the secret opening information of these commitments $s k_{\text {com }}=\left(s k_{c o m}^{W}, s k_{c o m}^{I}, s k_{c o m}^{k_{w}}\right) . P$ outputs a boolean value to the verifier, stating whether to accept the proof or not.

We can think a non-interactive designated verifier proof of an undeniable watermark. Such a scheme can diminish the difference between public watermark and undeniable watermark, in that it limits who can verify it without help from the prover, but does not necessary condition. We can use commit protocol. Therfore, the common three steps can be changed into one single step.

1. Generating and Proprocessing a Key/Data

- Generate prover Alice's secret key/public key pair : $x_{A}$ and $y_{A} \equiv$ $g^{x_{A}} \bmod p$.

- Generate verifier Bob's secret key/public key pair : $x_{B}$ and $y_{B} \equiv$ $g^{x_{B}} \bmod p$. (In this step, we use key generation function $G E N_{K E Y}$ ).

- Using hash function $h(\cdot)$, generate $m=h(I)$ on cover data $I$

- Alice selects $\omega, r, t \in Z_{q}$.

2. Generating and embedding a Watermark.

- The verifier sends hashed value $m$ to prover.

- The prover generate a watermark $W$ using his own secret key $x_{A}: W \equiv$ $m^{x_{A}} \bmod p$. (In this step, we use watermark generation function $G E N_{W}$ and we can tell the prover as a watermarker or signer).

- The prover embed this watermark $W$ into cover data $I$. Then the watermarked data $I^{W}$ will be generated. (In this step, we use embedding function $E$ ).

3. Constructing a Proof.

- The prover calculates $c=g^{\omega} y_{B}^{r} \bmod p, G \equiv g^{t} \bmod p, M \equiv m^{t} \bmod p$, and $d \equiv t+\left(x_{A} \cdot \omega\right) \bmod q$.

- And the prover sends commitment $(\omega, r, G, M, d)$ to the verifier.

4. Verifying a Proof.

- The verifier extract the embedded pseudo-watermark $W^{\prime}$ from the $I^{\prime}$. (In this step, we use detection function $D$ ).

- Using the commitment, the verifier calculates $c \equiv g^{\omega} y_{B}^{r} \bmod p$ and $G$. $y_{A}^{\omega} \equiv g^{t} \cdot g^{x_{A} \cdot \omega} \bmod p \equiv g^{d} \bmod p$.

- On the extracted pseudo-watermark, the (designated) verifier calculate $M \cdot W^{\omega} \equiv m^{d} \bmod p$.

\section{Discussion: Zero Knowledge Proof of Ownership}

Existing mechanism suffer from the watermark verification process: the disputing parties have to disclose information, which is necessary to detect the watermark, to the dispute-resolver. However, this information is in most cases also sufficient 
to remove attack and re-watermarking attack from this opened watermark(the disputed data). As a solution, zero-knowledge watermark verification eliminates this security risk, because it enables a prover to prove to an untrusted verifier that a certain watermark is present in watermarked data without revealing any information about the watermark, the reference data and the detection key. We can combine this properties with proof of owner procedures.

If ownership is challenged, both challenger and owner has to disclosed the proof of their ownership. So ownership is an issue, though people are seldom aware of it. If you are not able to prove you are the owner of something in the first place, you cannot ask for any payments concerning any kind of copyright. It is also important in digitized watermark system.

In proposed scheme, we suggest undeniable verification procedure. And it can be applicable to the proof of copyright or onwership of some resources by trusted third party $T$ or certificate authority $C A$. The copyright hodler $H$ can proof his own privilege as follows. We can considers $H$ to be the owner of a work $I^{\prime}$ if and only if the following conditions hold:

- $H$ has previously registered a new work $I^{\prime}$ on trusted third party $T$

$-I$ is similar to $I^{\prime}$ and

$-I^{\prime}$ is the first registered work to which $I$ is similar.

Therefore, we can construct ownership proofing function from the undeniable verification system. And the detailed mechanism can also be suggested by combining identification module with watermark verification. So, the real owner can be determined by similar verification procedure proposed in this study.

\section{Conclusions}

Digital watermarks have been proposed as the means for copyright protection of multimedia data. However, there are some problems in existing digital watermarking mechanism as the rightful ownerships cannot be resolved from diverse attacks such as re-watermarking. In this study, we solve multiple watermarking problem as we consider it by the designated verification process. In proposed system, if there are multiple watermarked image, the original owner can cooperate its verification process. So, in case that the examined labelpseudo-watermark can also be matched with the verifier's input message as a true-watermark, we can trust the verifierpseudo-owner as real true-owner by the proposed designated verification mechanism.

As there are some problems in existing digital watermarking mechanism, the rightful ownership cannot be resolved by existing watermarking schemes alone. Specially, some one can provide counterfeited watermark which can be performed on a watermarked image to allow multiple claims of rightful ownerships. Therefore, we proposed undeniable verification scheme in the watermarking process on network based secure image distribution. And using designated verifier proofing schemes, watermark can be verified by commitment methods. In the future, we can consider zero-knowledge based designated verification mechanism when the 
confirmer of a watermark verify the validity of the watermark owner without revealing the secret of true watermark information of originator.

\section{References}

1. Andre Adelsbach, Birgit Pfitzmann, Ahmad-Reza Sadeghi, "Proving Ownership of Digital Content", 3rd International Information Hiding Workshop (IHW '99), LNCS 1768, Springer-Verlag, 117-133, 1999.

2. Scott Craver, Nasir Memon, Boon-Lock Yeo, Minerva M. Yeung, "Resolving Rightful Ownerships with Invisible Watermarking Techniques: Limitations, Attacks, and Implications", IEEE Journal on Selected Areas in Communications 16/4 (1998) 573-586.

3. Andre Adelsbach, Ahmad-Reza Sadeghi, "Zero-Knowledge Watermark Detection and Proof of Ownership", 4th International Information Hiding Workshop (IHW '01), LNCS 2137, Springer-Verlag, 273-288, 2001.

4. Markus Jakobsson, Kazue Sako, and Russell Impagliazzo, "Designated verifier proofs and their applications", Advances in Cryptology - EUROCRYPT 96, LNCS 1070, Springer-Verlag, 143-154, 1996.

5. T. P. Pedersen, "Distributed provers with applications to undeniable signatures", Advances in Cryptology, Proceedings of Eurocrypt91, LNCS 547, pages 221-242, 1991.

6. T. ElGamal, "A Public Key Cryptosystem and a Signature Scheme based on Discrete Logarithm", IEEE Transactions on Information Theory, Vol. IT-30, No. 4, pp. 469-472, 1985.

7. I. B. D. J. Boyar, D. Chaum and T. P. Pedersen, "Convertible undeniable signatures", Advances in Cryptology, Proceedings of Crypto90, pages 189-205, 1991.

8. Alfred J. Menezed, Paul C. van Oorschot, Scott A. Vanstone (ed.), "Handbook of Applied Cryptography", CRC Press, 1996.

9. B. Schneier, "Applied Cryptography", Second Edition, Wiley, 1996.

10. D. Chaum, "Undeniable signatures", Advances in Cryptology, Proceedings of CRYPTO89, pages 212-216, 1989.

11. W. Diffie and M. Hellman, "New directions in cryptography", IEEE Transactions on Information Theory, IT-22(6):472-492, November 1976. 